\title{
Use of Diffusion Tensor MRI to Identify Early Changes in Diabetic Nephropathy
}

\author{
Lan Lu ${ }^{a} \quad J o h n R$. Sedor ${ }^{c, d}$ Vikas Gulani ${ }^{a}$ Jeffrey R. Schelling ${ }^{c, d}$ \\ Alicia O'Brien ${ }^{c, d}$ Chris A. Flask ${ }^{a, e}$ Katherine MacRae Dell ${ }^{b, d}$ \\ ${ }^{a}$ Department of Radiology, ${ }^{b}$ Department of Pediatrics and Rainbow Babies and Children's Hospital, \\ 'Department of Medicine, MetroHealth System Campus, ${ }^{d}$ CWRU Center for the Study of Kidney Disease and \\ Biology, and ${ }^{e}$ Department of Biomedical Engineering, Case Western Reserve University, Cleveland, Ohio, USA
}

\section{Key Words}

Diabetic nephropathy $\cdot$ Chronic kidney disease $\cdot$ Magnetic resonance imaging $\cdot$ Diffusion parameters $\cdot$ Diffusion tensor imaging

\begin{abstract}
Background/Aims: Currently available clinical indicators of kidney disease lack the sensitivity and/or specificity to identify early-stage diabetic nephropathy (DN). Quantitative diffusion magnetic resonance imaging (MRI), specifically diffusion tensor imaging (DTI), has been used to quantify pathophysiologic changes in other organs but has not been well studied in kidney diseases, including DN. The goal of this pilot study was to examine differences in kidney DTI parameters in diabetic subjects versus healthy controls. Methods: 16 diabetic and 5 healthy control subjects were recruited for this institutional review board-approved/Health Insurance Portability and Accountability Act-compliant study. Kidneys were scanned using DTI to generate apparent diffusion coefficient (ADC) and fractional anisotropy (FA) data. Mean cortical and medullary ADC and FA values were calculated by selecting multiple regions of interest. Diabetics were stratified by estimated glomerular filtration rate (eGFR) into 2 groups: eGFR $\geq 60(n=10)$ and eGFR $<60(n=6) \mathrm{ml} / \mathrm{min} / 1.73 \mathrm{~m}^{2}$.
\end{abstract}

Mean diffusion parameters and eGFRs were compared between these groups of diabetic subjects and healthy controls. Results: Medullary FA, ADC and cortical ADC values were significantly lower in diabetics with eGFR $<60$ compared to controls. Notably, both mean medullary FA and ADC were significantly lower in diabetics with eGFR $\geq 60$ compared to controls ( $p=0.001$ and $p=0.042$, respectively). For the study subjects in aggregate, medullary FA correlated significantly with eGFR $(R=0.69, p<0.01)$; the other diffusion parameters showed no significant correlations. Conclusions: This pilot study suggests that changes in medullary DTI assessments may serve as indicators of early DN. Further studies are needed to determine if these findings could serve as biomarkers to identify diabetics at risk of DN progression.

Copyright $\odot 2011$ S. Karger AG, Basel

\section{Introduction}

Diabetic nephropathy (DN) is the leading cause of end-stage renal disease (ESRD) in the United States. Its prevalence is increasing worldwide and will likely increase further as the epidemic of type 2 diabetes mellitus worsens [1]. Discovery of biomarkers that identify pa-

\section{KARGER}

๑) 2011 S. Karger AG, Basel

Fax +41 613061234 E-Mail karger@karger.ch www.karger.com www.karger.com/ajn
Katherine MacRae Dell, MD

Department of Research

MetroHealth Medical Center

2500 MetroHealth Drive, R457, Cleveland, OH 44109 (USA)

Tel. +1 216778 1156, E-Mail Katherine.Dell@ case.edu 
tients in the early stages of DN will permit targeted, intensified treatment to high-risk patient populations and facilitate clinical trials of new therapies. Although specific risk factors for progression to DN have been identified for diabetics as a whole, these do not reliably predict progression in individual diabetic patients [2]. Glomerular filtration rate (GFR)-estimating equations based on serum creatinine values lack precision and perform suboptimally in early-stage kidney disease; microalbuminuria may indicate early damage but regresses in a significant proportion of diabetics. Thus, neither traditional clinical marker of kidney disease has sufficient sensitivity and specificity to diagnose early $\mathrm{DN}$ (the point at which interventions are most likely to impact disease progression) or to predict progression to ESRD in individual diabetic patients.

Magnetic resonance imaging (MRI) techniques, specifically quantitative diffusion MRI methods, may offer the opportunity to noninvasively identify early renal damage. These methods have a number of advantages including the fact that they can be used to assess kidney structure and function without gadolinium chelates [38]. Standard quantitative diffusion-weighted imaging (DWI) methods provide an assessment of water's mobility in a given tissue, which is expressed as an apparent diffusion coefficient (ADC). A high ADC suggests a large amount of water movement, whereas a low ADC suggests restricted water movement. ADC values can vary based on the type of organ studied as well as the presence or absence of a given disease process. Cysts, for example, typically exhibit higher ADCs, whereas tissue fibrosis typically has lower ADCs [9-11].

Diffusion tensor imaging (DTI) is a diffusion MRI method that is more comprehensive than standard DWI. In DTI, information is obtained not only about the magnitude of water's mobility (ADC), but also its directionality and is expressed as fractional anisotropy (FA). The ability of DTI to quantify the directional nature of water movement makes it ideally suited for analyzing highly structured organs such as the kidney. For example, with DTI, a cyst has low FA (homogeneous water movement with no directionality), whereas highly structured tubules and vessels in the renal medulla are known to have a higher FA (water movement in one direction) $[7,8,11]$. Previous diffusion MRI studies in patients with chronic kidney disease (CKD) have been limited to DWI assessments of ADC $[8,11-13]$. DWI techniques have also been used to assess kidney changes in an experimental model of DN [14]. Although initial kidney DTI studies have been conducted in healthy human subjects [7], these tech- niques have not been evaluated as potential noninvasive assessments of DN. The goal of this pilot imaging study was to examine differences in kidney DTI parameters in a cohort of diabetics with a range of estimated GFRs (eGFRs) in comparison to healthy control subjects.

\section{Methods}

Subjects

Sixteen adult subjects with diabetes mellitus currently enrolled in the Renal Disease Progression Genes and Environmental Impact on Diabetic Nephropathy Study [15] were recruited for DTI scanning. This study is a longitudinal prospective study of diabetics with and without a family history of DN to identify genetic risk factors for progression to DN. All subjects, ages 40-65 years with eGFR $>25 \mathrm{ml} / \mathrm{min} / 1.73 \mathrm{~m}^{2}$ were eligible, except those with contraindications to an MRI (e.g. metal implants). For controls, the kidneys of 5 healthy, non-diabetic age-matched volunteers were also scanned. Subjects aged $40-65$ years with no known kidney disease, hypertension, heart disease or rheumatologic disease were recruited. eGFRs were calculated from serum creatinine measurements for all subjects based on the Chronic Kidney Disease Epidemiology Collaboration (CKD-EPI) equation [16]. This pilot imaging study was conducted according to approved Institutional Review Board, Health Insurance Portability and Accountability Act-compliant protocols.

\section{Renal MRI Image Acquisition}

Each subject was scanned in a supine position with a Siemens Espree 1.5T MRI scanner (70 $\mathrm{cm}$ bore) to accommodate the diabetic subjects. Volume status was not assessed or controlled at the time of imaging. Spine array (posterior) and body array (anterior) receiver coils were used to maximize image uniformity. Following initial localizer scans, coronal T2-weighted MR images (Half-Fourier Acquisition Single-shot Turbo spin Echo (HASTE), respiratory-triggered, $\mathrm{TR} / \mathrm{TE}=1,000 / 72 \mathrm{~ms}$, slice thickness $=6 \mathrm{~mm}, 10$ slices, $\mathrm{FOV}=360 \times 210 \mathrm{~mm}$, partial Fourier factor 5/8, 1 average) were obtained to delineate medullary and cortical kidney regions. The HASTE acquisition was respiratory-gated to ensure accurate image co-registration with the respiratory-gated diffusion acquisitions described below. A respiratory-gated, single-shot diffusion tensor imaging - echo planar imaging (DTI-EPI) acquisition was then used to acquire diffusion-weighted images of the left and right kidneys $\left(\mathrm{b}=0\right.$ and $400 \mathrm{~s} / \mathrm{mm}^{2}, 6$ directions + null, TR/TE $=$ $2,000 \mathrm{~ms} / 75 \mathrm{~ms}$, imaging slice thickness $=6 \mathrm{~mm}, 10$ imaging slices/subject). Six imaging averages were acquired to obtain images with a sufficient signal-to-noise ratio (SNR) to ensure an accurate FA assessment. Fat suppression and slab suppression radiofrequency pulses were also added to limit chemical shift and motion artifacts, respectively. The total scan time for each DTI acquisition was 4-6 min, depending on each subject's respiration rate.

\section{DTI Calculations and Region of Interest Analysis}

All MRI images were exported and processed offline in Matlab (The Mathworks, Natick, Mass.). Image maps representing the $\mathrm{ADC}$ and FA at each imaging pixel were obtained from the diffusion-weighted images (6 directions + null) according to well-established procedures [3]. 
Table 1. Clinical features of diabetic subjects

\begin{tabular}{|c|c|c|c|c|c|c|c|c|c|}
\hline Sex & Race & $\begin{array}{l}\text { Age } \\
\text { years }\end{array}$ & $\begin{array}{l}\text { eGFR, } \mathrm{ml} / \\
\mathrm{min} / 1.73 \mathrm{~m}^{2}\end{array}$ & $\begin{array}{l}\text { Duration of } \\
\text { DM, years }\end{array}$ & HTN & $\begin{array}{l}\text { Reti- } \\
\text { nopathy }\end{array}$ & Smoking & $\begin{array}{l}\text { Urine albumin } \\
\mathrm{mg} / \mathrm{gm} \\
\text { creatinine }\end{array}$ & $\begin{array}{l}\text { HgbAlC } \\
\%\end{array}$ \\
\hline Female & $\mathrm{AA}$ & 51 & 110 & 19 & $\mathrm{~N}$ & $\mathrm{~N}$ & $\mathrm{~N}$ & 5 & 7.6 \\
\hline Female & AA & 45 & 108 & 29 & $\mathrm{Y}$ & $\mathrm{N}$ & $\mathrm{N}$ & 11 & 8 \\
\hline Female & $\mathrm{C}$ & 52 & 103 & 17 & $\mathrm{Y}$ & $\mathrm{N}$ & $\mathrm{N}$ & 5 & 9.1 \\
\hline Female & $\mathrm{C}$ & 58 & 88 & 29 & $\mathrm{Y}$ & $\mathrm{N}$ & $\mathrm{N}$ & 79 & 10 \\
\hline Female & $\mathrm{AA}$ & 62 & 87 & 24 & $\mathrm{Y}$ & $\mathrm{N}$ & $\mathrm{N}$ & 252 & 10.7 \\
\hline Female & $\mathrm{C}$ & 61 & 85 & 11 & $\mathrm{Y}$ & $\mathrm{N}$ & $\mathrm{N}$ & 34 & 6.2 \\
\hline Female & $\mathrm{AA}$ & 63 & 81 & 20 & $\mathrm{Y}$ & $\mathrm{N}$ & $\mathrm{N}$ & 25 & 11.3 \\
\hline Female & $\mathrm{AA}$ & 53 & 73 & 31 & $\mathrm{Y}$ & $\mathrm{Y}$ & $\mathrm{N}$ & 179 & 8.5 \\
\hline Male & $\mathrm{C}$ & 58 & 68 & 45 & $\mathrm{Y}$ & $\mathrm{N}$ & $\mathrm{N}$ & 6 & 6.9 \\
\hline Female & $\mathrm{AA}$ & 61 & 63 & 10 & $\mathrm{Y}$ & $\mathrm{N}$ & $\mathrm{N}$ & 9 & 6.5 \\
\hline Female & $\mathrm{C}$ & 57 & 58 & 13 & $\mathrm{Y}$ & $\mathrm{Y}$ & $\mathrm{N}$ & 1,030 & 6.3 \\
\hline Female & $\mathrm{C}$ & 57 & 58 & 32 & $\mathrm{~N}$ & $\mathrm{Y}$ & $\mathrm{N}$ & 74 & 12.2 \\
\hline Female & $\mathrm{AA}$ & 65 & 55 & 23 & $\mathrm{Y}$ & $\mathrm{N}$ & $\mathrm{N}$ & 12 & 7.2 \\
\hline Female & $\mathrm{AA}$ & 48 & 39 & 36 & $\mathrm{Y}$ & $\mathrm{N}$ & $\mathrm{N}$ & 114 & 10.3 \\
\hline Female & $\mathrm{AA}$ & 64 & 29 & 30 & $\mathrm{Y}$ & $\mathrm{N}$ & $\mathrm{N}$ & 18 & 7 \\
\hline Male & $\mathrm{AA}$ & 59 & 27 & 29 & $\mathrm{Y}$ & $\mathrm{Y}$ & $\mathrm{Y}$ & 725 & 8 \\
\hline
\end{tabular}

$\mathrm{AA}=$ African-American; $\mathrm{C}=$ Caucasian; $\mathrm{HTN}=$ hypertension; $\mathrm{Y}=$ yes; $\mathrm{N}=$ no.

\begin{abstract}
A region of interest (ROI) analysis of the ADC and FA maps was performed to obtain measures of mean cortical and medullary $\mathrm{ADC}$ and FA for each subject. Four coronal imaging slices nearest to the renal hilum of the left and right kidneys of each subject were selected for the ROI analysis. ROIs were selected by an expert rater (L.L.) trained by a board-certified radiologist and a nephrologist. The rater was blinded to the eGFR results during the ROI analysis. Medullary and cortical ROI selections were obtained in the HASTE images with high corticomedullary contrast and high anatomic detail. For each imaging slice, 4 medullary and 4 cortical ROIs were selected to ensure adequate sampling. A minimum of 10 pixels/ROI was also incorporated to limit the effects of noise. The ROIs selected in the HASTE images were directly applied to the corresponding (and coregistered) ADC and FA maps to obtain mean cortical and medullary ADC and FA assessments. Overall mean ADC and FA for each subject were obtained by averaging overall ROIs for all 4 imaging slices and both left and right kidneys (i.e. 32 total medullary ROIs and 32 total cortical ROIs).
\end{abstract}

\section{Statistical Analysis}

Mean medullary and cortical diffusion parameters (ADC, FA) from the ROI analysis as well as eGFRs of diabetic and healthy control subjects were compared using two-tailed Student's t test. The diabetic subjects were analyzed as 2 separate groups: (1) GFR $<60(\mathrm{n}=6)$, and $(2)$ eGFR $\geq 60(\mathrm{n}=10) \mathrm{ml} / \mathrm{min} / 1.73 \mathrm{~m}^{2}$. The 2 diabetic groups were analyzed separately to identify potential DTI differences in these subject groups compared to healthy control subjects. Mean cortical and medullary ADC and FA were also plotted as a function of eGFR for all subjects and Pearson correlation coefficients $(\mathrm{R})$ were determined from a least squared error fit to a linear model.

\section{Results}

Clinical features of the 16 diabetic subjects are shown in table 1. Diabetic subjects had an eGFR range of 27-110 (mean: $71 \pm 26) \mathrm{ml} / \mathrm{min} / 1.73 \mathrm{~m}^{2}$, an age range of 51-66 (mean: $57 \pm 6$ ) years and diabetes duration of 10-45 (mean: $25 \pm 10)$ years. Male $(\mathrm{n}=2)$ and female $(\mathrm{n}=14)$ subjects as well as African-American $(\mathrm{n}=10)$ and Caucasian $(n=6)$ subjects were included. Albuminuria was highly variable $(5-1,030 \mathrm{mg} / \mathrm{g}$ creatinine).

Control subjects ( 3 females/2 males) were otherwise healthy individuals with no known history of kidney disease, systemic disease, hypertension or proteinuria. The 5 healthy controls had eGFR values of 76, 86, 90, 93, 102 (mean: $90 \pm 9$ ) $\mathrm{ml} / \mathrm{min} / 1.73 \mathrm{~m}^{2}$ and an age range of $41-58$ (mean: $48 \pm 6$ ) years. Figure 1 illustrates a representative standard T2-weighted HASTE image. These images were used to select the cortical and medullary ROIs for the quantitative analysis of the ADC and FA maps as described above. Figure 2 shows representative ADC and FA maps (color representations of $\mathrm{ADC}$ and FA values within each kidney) for a healthy subject (eGFR $=90 \mathrm{ml} / \mathrm{min} / 1.73$ $\mathrm{m}^{2}$ ) and 3 diabetic subjects (eGFRs $=110,103$ and $29 \mathrm{ml} /$ $\mathrm{min} / 1.73 \mathrm{~m}^{2}$ ). Red hues represent higher ADC or FA values, whereas blue hues represent lower values. Findings for the left and right kidney were similar for all groups 
1



Fig. 1. a Coronal T2-weighted MRI images of a control kidney. b Representative cortical and medullary ROIs are overlaid onto representative T2-weighted HASTE images. Fig. 2. Comparisons of diffusion MRI parameters in a healthy control and in diabetics with different levels of estimated renal function. Imaging data (ADC and FA diffusion maps) are shown for a healthy control subject (first panel), 2 diabetic subjects with normal eGFRs (2nd and 3rd panel) and a diabetic subject with more advanced disease (4th panel). Medullary FA for 1 of the diabetic subjects with a normal eGFR is similar to that of the healthy control, with prominent areas of high FA evident. In contrast, medullary FA is decreased for the other diabetic with a normal eGFR as well as the diabetic subject with advanced CKD.
2

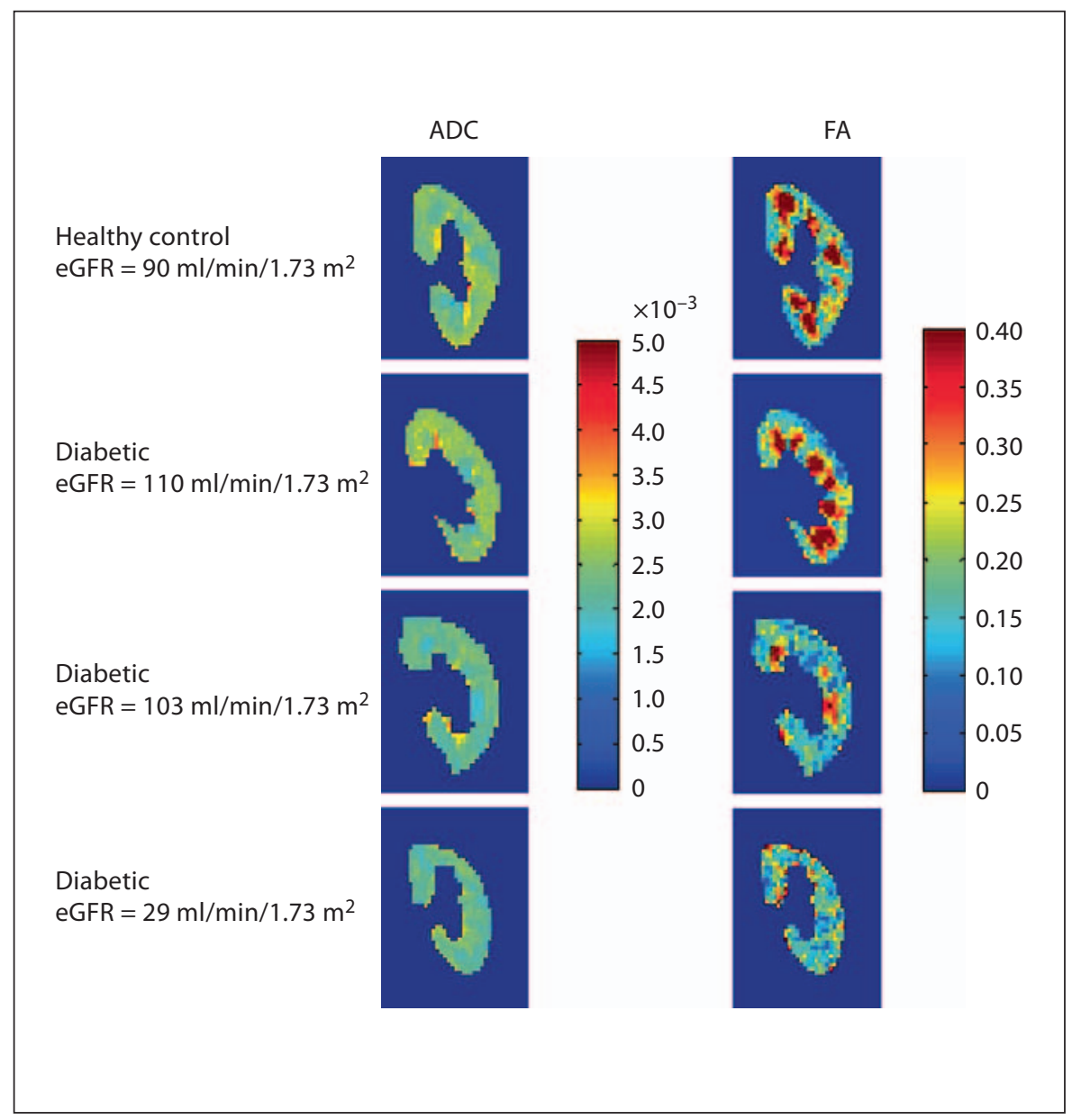

studied (data not shown), so only 1 representative kidney is shown for each subject. Note that high SNR diffusionweighted images were generated for each subject with minimal artifacts associated with respiratory motion.

Distinct hyperintense medullary regions readily distinguishable from cortical areas are clearly visible in the FA maps for the healthy subject (1st panel) and the diabetic subject with an eGFR of $110 \mathrm{ml} / \mathrm{min} / 1.73 \mathrm{~m}^{2}$ (2nd panel). In contrast, for the other 2 diabetic subjects ( $3 \mathrm{rd}$ and 4th panels), medullary regions with high FA values were much less prominent. Note the similarity in the FA results for these 2 diabetic subjects despite the large difference in eGFRs (103 vs. $29 \mathrm{ml} / \mathrm{min} / 1.73 \mathrm{~m}^{2}$ ).

Table 2 summarizes the medullary and cortical ADC and FA values obtained from the ROI analysis for diabetic subjects with eGFR $<60 \mathrm{ml} / \mathrm{min} / 1.73 \mathrm{~m}^{2}(\mathrm{n}=6)$, eGFR $\geq 60(n=10)$ and healthy controls $(n=5)$. Not unexpectedly, the mean medullary FA, cortical FA and cortical ADC values for diabetics with eGFR $<60$ were sig- nificantly lower than those of healthy controls. This is consistent with published data showing lower ADC values in subjects with CKD compared to controls [8, 12, 17]. Interestingly, mean medullary FA and medullary ADC of the diabetics with relatively intact kidney function (eGFR $\geq 60$ ) were also significantly lower than those of healthy controls ( $p=0.001$ for FA; $p=0.042$ for ADC). These lower medullary FA and ADC values were observed despite the fact that no significant differences in mean eGFR were seen between the 2 groups (healthy controls: $90 \pm 9$ (range 76-102); diabetics with eGFR $\geq 60: 87 \pm$ 16 (range $63-110) \mathrm{ml} / \mathrm{min} / 1.73 \mathrm{~m}^{2}$ ). As illustrated in figure 3, a significant correlation was observed between eGFR and medullary FA $(r=0.69$, $p<0.01)$ for all subjects. A similar significant correlation was observed for diabetics alone $(r=0.66, \mathrm{p}<0.01)$. In contrast, correlations between eGFR and the other three imaging parameters (i.e. cortical FA, medullary and cortical ADC) for all subjects or diabetics alone were not significant. 
Table 2. Summary of imaging data for all subjects

\begin{tabular}{|c|c|c|c|c|c|}
\hline & $\begin{array}{l}\mathrm{eGFR}, \mathrm{ml} / \\
\mathrm{min} / 1.73 \mathrm{~m}^{2}\end{array}$ & $\begin{array}{l}\text { Medullary } \\
\text { FA }\end{array}$ & $\begin{array}{l}\text { Cortical } \\
\text { FA }\end{array}$ & $\begin{array}{l}\text { Medullary } \\
\text { ADC }\end{array}$ & $\begin{array}{l}\text { Cortical } \\
\text { ADC }\end{array}$ \\
\hline \multirow{3}{*}{$\begin{array}{l}\text { Diabetic eGFR }<60 \\
(\mathrm{n}=6) \\
\text { Diabetic eGFR } \geq 60 \\
(\mathrm{n}=10) \\
\text { Healthy controls } \\
(\mathrm{n}=5)\end{array}$} & $44 \pm 15^{\mathrm{a}}$ & $0.230 \pm 0.028^{b}$ & $0.180 \pm 0.028$ & $2.086 \pm 0.177^{\mathrm{c}}$ & $2.211 \pm 0.150^{c}$ \\
\hline & $87 \pm 16$ & $0.252 \pm 0.029^{b}$ & $0.172 \pm 0.024$ & $2.160 \pm 0.202^{\mathrm{c}}$ & $2.330 \pm 0.283$ \\
\hline & $90 \pm 9$ & $0.298 \pm 0.013$ & $0.190 \pm 0.013$ & $2.338 \pm 0.104$ & $2.507 \pm 0.136$ \\
\hline
\end{tabular}

${ }^{\mathrm{a}} \mathrm{p}<0.0005 \mathrm{vs}$. healthy controls or diabetics with eGFR $\geq 60 \mathrm{ml} / \mathrm{min} / 1.73 \mathrm{~m}^{2} .{ }^{\mathrm{b}} \mathrm{p}<0.005 \mathrm{vs}$. healthy controls. ${ }^{\mathrm{c}} \mathrm{p}<0.05 \mathrm{vs}$. healthy controls. ${ }^{\mathrm{d}} \mathrm{p}<0.0001$ vs. healthy controls.

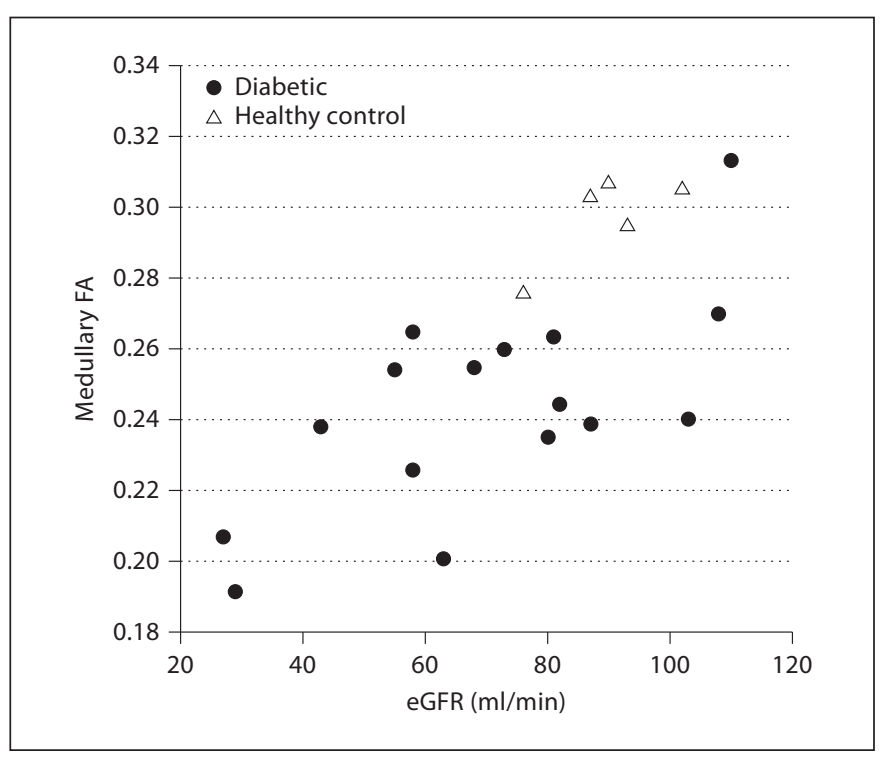

Fig. 3. Medullary FA and eGFR in diabetic and normal subjects. The dot plot shows medullary FA plotted as a function of eGFR for both diabetic $(\bullet)$ and control subjects $(\triangle)$. A highly significant Pearson correlation $(\mathrm{r}=0.69, \mathrm{p}<0.01)$ was observed between medullary FA and eGFR. Correlations between the other imaging parameters (cortical FA, medullary ADC and cortical ADC) and eGFR were not significant.

\section{Discussion}

In this pilot study using quantitative DTI techniques, we identified significantly lower mean medullary FA values among diabetics with relatively intact renal function (eGFR $\geq 60 \mathrm{ml} / \mathrm{min} / 1.73 \mathrm{~m}^{2}$ ) compared to healthy control subjects. These medullary FA differences were observed despite a lack of significant differences in eGFRs between these 2 groups. Medullary ADC values were also significantly lower in this subset of diabetic subjects compared to control subjects. Cortical FA and ADC were not significantly different between these 2 groups. In addition, diabetics with moderate to severe renal function impairment (eGFR $<60 \mathrm{ml} / \mathrm{min} / 1.73 \mathrm{~m}^{2}$ ) exhibited lower medullary FA and medullary and cortical ADCs compared to healthy control subjects. Medullary FA was the only one of the four imaging parameters (medullary FA and $\mathrm{ADC}$, cortical FA and ADC) to demonstrate a significant correlation with eGFR.

Previous diffusion-weighted MRI studies have shown renal $\mathrm{ADC}$ values are reduced in a variety of acute and chronic kidney diseases $[8,12,18,19]$. In contrast to previous studies, however, the current study utilized DTI techniques and focused specifically on diabetics, including diabetic subjects with relatively intact renal function (eGFR $\geq 60 \mathrm{ml} / \mathrm{min} / 1.73 \mathrm{~m}^{2}$ ). While the numbers of subjects in the current pilot study are relatively small, our findings suggest that both medullary FA and ADC may identify early changes in diabetics, which could indicate early DN. Longitudinal studies in larger numbers of diabetic subjects are necessary to confirm this hypothesis and determine the sensitivity and specificity of these two MRI parameters in identifying early DN changes.

The pathophysiologic process underlying the observed decreases in mean FA and ADC in the diabetic subjects is not well understood. Several potential mechanisms can be postulated, and more than one may be involved. These include reduced tubular flow rate, tubular damage, and vascular abnormalities, including changes upstream of the vasa recta. However, further clinical and preclinical studies are needed to help distinguish between tubular and vascular changes and to better understand the rela- 
tionship of these observations to the pathophysiologic changes in early-stage DN. Decreased medullary FA may also characterize all-cause early CKD, rather than being a specific feature of DN. Additional studies in non-diabetic CKD subjects will be necessary to answer this question.

This pilot study using DTI techniques to assess renal physiology in diabetics has several limitations. Most importantly, the number of subjects is relatively small and these compelling initial results will need to be verified in a larger cohort of diabetics. Further validation of these initial results would also benefit from direct measurement of GFR rather than estimates based on serum creatinine. This is true for both the diabetic and healthy controls. Of note, 1 healthy control (without any significant medical issues) had an eGFR of $76 \mathrm{ml} / \mathrm{min} / 1.73 \mathrm{~m}^{2}$. While we suspect that this represents the known inaccuracy of eGFR estimates in patients with eGFR $\geq 60 \mathrm{ml} /$ $\mathrm{min} / 1.73 \mathrm{~m}^{2}$ [16], we cannot definitively say that this subject was truly 'healthy'. Longitudinal DTI assessments may also determine if diabetics with high eGFRs and low medullary ADC/FA are susceptible to progression to clinically evident DN. In addition, volume status was not formally assessed or controlled for and this could have had an impact on tubular flow. The diffusion MRI techniques could also be expanded to more rigorously understand the impact of disease progression on the diffusion parameters. Previous studies have shown that renal ADC is more accurately measured using a bi-exponential model of diffusion to provide separate estimates of diffusion and perfusion [11]. The kidney DTI results shown here use an alternative approach focusing on the directionality (FA) rather than the magnitude of renal diffusion. A more comprehensive approach to the study of renal diffusion in these subjects would be to apply the bi-exponential model (i.e. multiple b values) in combination with a DTI acquisition. With this approach, rigorously accurate assessments of ADC and FA could be obtained while still separating out the effects of tissue perfusion. However, this approach could require 1-2 h of acquisition time which may not be practical for trials in human subjects.

In summary, the results of this pilot study suggest that medullary FA and ADC, quantified by kidney DTI, may be potential imaging biomarkers for $\mathrm{DN}$. The successful development of radiologic measures to identify early-stage DN would enable clinical intervention at a time when the disease may be effectively treated and could facilitate clinical trials of new therapeutic strategies. These data suggest that quantitative DTI certainly merits study in diabetic cohorts to assess validity in staging and predicting patients at risk of progression to ESRD.

\section{Acknowledgements}

This study was presented in abstract form at the 2010 Annual Meeting of the American Society of Nephrology. This publication was made possible by the Case Western Reserve University/Cleveland Clinic CTSA grant number UL1 RR024989 from the National Center for Research Resources (NCRR), a component of the National Institutes of Health and NIH roadmap for Medical Research. Its contents are solely the responsibility of the authors and do not necessarily represent the official view of NCRR or NIH. Additional funding support was provided by NIH/NIDDK 5R01DK059997, Renal Disease Progression in African Americans; and NIH/NIDDK 5U01DK057329, Case FIND Participating Investigative Center. The authors would also like to thank Dr. John O'Toole for a critical review of the manuscript.

\section{References}

1 Wild S, Roglic G, Green A, Sicree R, King H: Global prevalence of diabetes: estimates for the year 2000 and projections for 2030. Diabetes Care 2004;27:1047-1053.

2 Retnakaran R, Cull CA, Thorne KI, Adler AI, Holman RR: Risk factors for renal dysfunction in type 2 diabetes: UK Prospective diabetes study 74. Diabetes 2006;55:18321839.

-3 Basser PJ, Mattiello J, LeBihan D: Estimation of the effective self-diffusion tensor from the NMR spin-echo. J Magn Reson B 1994;103: 247-254.
4 Chandarana H, Lee VS: Renal functional MRI: are we ready for clinical application? AJR Am J Roentgenol 2009;192:1550-1557.

5 Huang AJ, Lee VS, Rusinek H: MR imaging of renal function. Radiol Clin North Am 2003;41:1001-1017.

6 Kim S, Naik M, Sigmund E, Taouli B: Diffusion-weighted MR imaging of the kidneys and the urinary tract. Magn Reson Imaging Clin N Am 2008;16:585-596.

$\checkmark 7$ Ries M, Jones RA, Basseau F, Moonen CT, Grenier N: Diffusion tensor MRI of the human kidney. J Magn Reson Imaging 2001;14: 42-49.
-8 Thoeny HC, De Keyzer F, Oyen RH, Peeters RR: Diffusion-weighted MR imaging of kidneys in healthy volunteers and patients with parenchymal diseases: initial experience. Radiology 2005;235:911-917.

-9 Taouli B, Tolia AJ, Losada M, Babb JS, Chan ES, Bannan MA, Tobias H: Diffusionweighted MRI for quantification of liver fibrosis: preliminary experience. AJR Am J Roentgenol 2007;189:799-806. 
$>_{10}$ Annet L, Peeters F, Abarca-Quinones J, Leclercq I, Moulin P, Van Beers BE: Assessment of diffusion-weighted MR imaging in liver fibrosis. J Magn Reson Imaging 2007; 25:122-128.

11 Zhang JL, Sigmund EE, Chandarana H, Rusinek H, Chen Q, Vivier PH, Taouli B, Lee VS: Variability of renal apparent diffusion coefficients: limitations of the monoexponential model for diffusion quantification. Radiology 2010;254:783-792.

- 12 Xu X, Fang W, Ling H, Chai W, Chen K: Diffusion-weighted MRI imaging of kidneys in patients with chronic kidney disease: initial study. Eur Radiol 2010;20:978-983.

-13 Xu Y, Wang X, Jiang X: Relationship between the renal apparent diffusion coefficient and glomerular filtration rate: preliminary experience. J Magn Reson Imaging 2007;26:678681.
4 Ries M, Basseau F, Tyndal B, Jones R, Deminiere C, Catargi B, Combe C, Moonen CW, Grenier N: Renal diffusion and bold MRI in experimental diabetic nephropathy. Blood oxygen level-dependent. J Magn Reson Imaging 2003;17:104-113.

15 Bleyer AJ, Sedor JR, Freedman BI, O’Brien A, Russell GB, Graley J, Schelling JR: Risk factors for development and progression of diabetic kidney disease and treatment patterns among diabetic siblings of patients with diabetic kidney disease. Am J Kidney Dis 2008 51:29-37.
6 Levey AS, Stevens LA, Schmid CH, Zhang YL, Castro AF 3rd, Feldman HI, Kusek JW, Eggers P, Van Lente F, Greene T, Coresh J: A new equation to estimate glomerular filtration rate. Ann Intern Med 2009;150:604612.

17 Wu M, Lin Y, Shieh C, Wan Y, Yen TH, Ng K, Wai Y, Wang J: Measuring anisotropic diffusion in kidney using MRI. Acad Radiol 2011; 18:1168-1174.

18 Namimoto T, Yamashita Y, Mitsuzaki K, Nakayama Y, Tang Y, Takahashi M: Measurement of the apparent diffusion coefficient in diffuse renal disease by diffusion-weighted echo-planar MR imaging. J Magn Reson Imaging 1999;9:832-837.

$>19$ Thoeny HC, De Keyzer F: Diffusion-weighted MR imaging of native and transplanted kidneys. Radiology 2011;259:25-38. 